\title{
Anurofauna de remanescentes de floresta Atlântica do município de São José do Barreiro, estado de São Paulo, Brasil
}

\author{
Herbert Serafim ${ }^{1,2,4}$, Susan Ienne ${ }^{2}$, Paulo José Pyles Cicchi ${ }^{3} \&$ Jorge Jim $^{3}$ \\ ${ }^{1}$ Laboratório de Herpetologia, Instituto Butantan, \\ Av. Doutor Vital, 1500, CEP 05503-900, São Paulo, SP, Brasil \\ ${ }^{2}$ Departamento de Genética e Biologia Evolutiva, Instituto de Biociências, \\ Universidade de São Paulo - USP, Rua do Matão, Travessa 14, 321, \\ CEP 05508-900, São Paulo, SP, Brasil \\ ${ }^{3}$ Departamento de Zoologia, Instituto de Biociências, Universidade Estadual Paulista - UNESP, \\ Distrito de Rubião Júnior, s/n, CEP 18618-000, Botucatu, SP, Brasil \\ ${ }^{4}$ Autor para correspondência: Herbert Serafim,e-mail: hserafim@usp.br
}

SERAFIM, H., CICCHI, P.J.P., IENNE, S. \& JIM, J. 2008. Anurans of remnants of Atlantic forest of São José do Barreiro municipality, São Paulo State, Brazil. Biota Neotrop. 8(2): http://www.biotaneotropica.org.br/ v8n2/en/abstract?article+bn01008022008.

\begin{abstract}
The municipality of São José do Barreiro (SP) located in the Atlantic Forest Domain, at elevations between 480 and $2088 \mathrm{~m}$ above sea level, exhibit ample topografic and climatic complexity that result in the presence of severals phytophysionomies with High Mountain Grasslands, Seasonal Semideciduous Forest, Tropical Rainforest, and Araucaria Forest. The aim of this study was to verify the anuran species richness in two different forest habitats in this area, one in a Seasonal Semideciduous Forest and the other in a Tropical Rainforest. Animals were collected by active search from April 2004 to December 2006. In the studied period, we registered 35 anuran species in nine families. The anuran richness observed in the studied region is similar to other localities considered preserved, as the Estação Ecológica Juréia-Itatins (Peruíbe, SP) and Serra do Japi (Jundiaí-SP). The similarity in species composition among seven localities in Atlantic Forest in São Paulo state was associated to the vegetal types of the studied areas. The ample altitude variation, the presence of different types of vegetation, and the little knowledge of the local fauna in this region, make the surveys very important to support future studies on species conservation.
\end{abstract}

Keywords: amphibia, conservation, species richness, serra da Bocaina.

SERAFIM, H., CICCHI, P.J.P., IENNE, S. \& JIM, J. 2008. Anurofauna de remanescentes de floresta Atlântica do município de São José do Barreiro, estado de São Paulo, Brasil. Biota Neotrop. 8(2): http://www. biotaneotropica.org.br/v8n2/pt/abstract?article+bn01008022008.

Resumo: O município de São José do Barreiro (SP), localizado no domínio da Floresta Atlântica, em altitudes que oscilam entre 480 e $2088 \mathrm{~m}$, apresenta grande complexidade topográfica e climática, que resulta na presença de inúmeras fitofisionomias, como os Campos de altitude, Floresta Estacional Semidecidual e Florestas Ombrófilas Densa e Mista. O objetivo deste estudo foi verificar a riqueza de espécies de anuros em duas áreas deste município: uma de Floresta Estacional Semidecidual e outra de Floresta Ombrófila Densa. Foram realizadas buscas ativas diurnas e noturnas, entre abril de 2004 e dezembro de 2006. O inventário resultou no registro de 35 espécies de anuros de nove famílias. A riqueza de espécies observada na área de estudo é similar a de outras localidades consideradas preservadas como a Estação Ecológica Juréia-Itatins (Peruíbe, SP) e a Serra do Japi (Jundiaí-SP). A similaridade na composição de espécies de sete localidades no Estado de São Paulo foi associada à fisionomia da vegetação das áreas amostradas. A grande variação de altitude, a presença de diferentes formações vegetais e o pouco conhecimento da fauna local, torna o inventário da sua anurofauna de suma importância para estudos futuros de conservação destas espécies.

Palavras-chave: anfíbios, conservação, riqueza de espécies, serra da Bocaina. 


\section{Introdução}

A Mata Atlântica é um dos Domínios fitogeográficos mais ricos em diversidade biológica do planeta, sendo considerado um dos vinte e cinco hotspots de biodiversidade, devido ao elevado grau de endemismos e alto grau de ameaça (Myers et al. 2000). No sudeste brasileiro a fragmentação da Mata Atlântica encontra-se em estágio avançado e a preservação de suas áreas remanescentes constitui um dos maiores problemas de conservação do País (MMA/IBAMA 2004, SP: SMA/IF 2005, Tabarelli et al. 2005). As áreas de Mata Atlântica ainda hoje bem preservadas estão localizadas basicamente em escarpas muito íngremes ou em altitudes elevadas, onde a prática agrícola ou madeireira torna-se inviável, além de outras poucas áreas de preservação ambiental (Fundação SOS Mata Atlântica 1998).

Em São Paulo, a devastação reduziu para pouco mais de $7 \%$ (1.731.472 ha) a área coberta por florestas naturais que ocupavam $81,8 \%$ do território paulista (20.450.000 ha; Fundação SOS Mata Atlântica \& INPE 1993). O processo de substituição da vegetação nativa, principalmente da cobertura florestal, em função da expansão das atividades agropecuárias e das malhas urbanas, assim como do extrativismo, ocasionou, no interior do estado, a fragmentação das florestas, resultando na formação de pequenas manchas ou de fragmentos isolados (Fonseca \& Rodrigues 2000).

Atualmente são conhecidas no mundo cerca de 5500 espécies de anfíbios anuros (AmphibiaWeb 2008). O Brasil possui a maior riqueza e a segunda maior diversidade de espécies de anfíbios (Mittermeier et al. 1997), com 817 espécies conhecidas, sendo 789 pertencentes à Ordem Anura (SBH 2008). Das 280 espécies de anfíbios da Mata Atlântica, 253 são endêmicas (Myers et al. 2000).

Embora o declínio mundial de anfíbios tenha sido evidenciado desde 1980, relativamente pouco se conhece a respeito do status de suas populações nos países da América do Sul (Silvano \& Segalla 2005). Dentre os entraves estão a falta de dados relacionados à distribuição de espécies e dinâmica das populações e a incipiência de conhecimento sobre sua diversidade (Young et al. 2001, Eterovick et al. 2005). Têm-se verificado extinções de espécies e mudanças no tamanho de inúmeras populações de anuros em diversos países da região Neotropical (Young et al. 2001). No Brasil, diversos estudos têm buscado compreender as razões dos declínios populacionais de anfíbios e da extinção de espécies, porém tais abordagens estão concentradas na região sudeste (e.g. Heyer et al. 1988, Izecksohn \& Carvalho-e-Silva 2001, Papp \& Papp 2000, Pombal Jr. \& Haddad 1999). A lista da anurofauna brasileira ameaçada de extinção apresenta 15 espécies nas categorias de ameaça e uma espécies considerada extinta, todas da Mata Atlântica (Silvano \& Segalla 2005).

Apesar da concentração de estudos nesta região, a anurofauna da Mata Atlântica é pouco conhecida devido à exigüidade de estudos a médio e longo prazo e à falta de levantamentos faunísticos em diferentes regiões (Haddad \& Sazima 1992, Pombal \& Gordo 2004, Silvano \& Segalla 2005).

Paralelamente ao pouco conhecimento da anurofauna da Mata Atlântica, existe o problema da velocidade de destruição dos ambientes naturais por ação antrópica (Heyer et al. 1990, Silvano \& Segalla 2005). As modificações produzidas pela ação do homem conduzem a uma uniformização do ambiente e redução no número de espécies com o aumento da abundância de outras, e/ou à invasão desses ambientes por espécies generalistas dos hábitats abertos vizinhos (Jim 1980, Haddad \& Prado 2005).

Tendo em vista a carência de informações sobre diversidade e distribuição da anurofauna brasileira e objetivando ampliar o conhecimento a respeito do grupo em uma região nunca antes estudada, neste estudo realizamos o levantamento da anurofauna de duas áreas do Domínio da Floresta Atlântica localizadas na região do Vale do Paraíba paulista no município de São José do Barreiro, e comparamos a composição desta anurofauna com a de outras localidades do estado de São Paulo

\section{Material e Métodos}

\section{1. Área de estudo}

O estudo foi desenvolvido na região do Vale do Paraíba (SP), área que possui um histórico de devastação antrópica associado principalmente aos ciclos da cana de açúcar, do café e, nas últimas décadas, ao da agropecuária (Dean 1997). A bacia Hidrográfica do rio Paraíba do Sul e Serra da Mantiqueira, com área total de 1.503.800 ha, apresenta apenas 329.177 ha de vegetação natural remanescente, $21,9 \%$ da sua superfície (SP:SMA/IF 2005). Embora seja uma das Bacias com maiores índices de vegetação remanescente do Estado de São Paulo, apresenta-se bastante fragmentada, com um total de 15.067 fragmentos (81,6\%), com superfície de até 20 ha (SP:SMA/IF 2005). O município de São José do Barreiro (S.J.Barreiro) localiza-se próximo à divisa entre os estados de São Paulo e Rio de Janeiro. Abrange desde áreas com baixas altitudes na região do Vale do Paraíba, em torno de $500 \mathrm{~m}$ de altitude, até áreas com altas altitudes na Serra da Bocaina, tendo como ponto culminante o Pico do Tira Chapéu, com $2.088 \mathrm{~m}$ de altitude. Neste município, encontram-se remanescentes de Campos de Altitude, Floresta Estacional Semidecidual, Floresta Ombrófila Mista e Floresta Ombrófila Densa, sensu Veloso et al. (1991). Partes destes remanescentes integram uma importante Unidade de Conservação, o Parque Nacional da Serra da Bocaina, com 104.000 ha, que representa a maior área de Floresta Atlântica protegida no país e também o maior Parque Nacional da região sudeste (MMA/IBAMA 2004).

O inventário de espécies de anuros foi realizado em duas áreas, com diferentes formações florestais, distantes cerca de $8 \mathrm{~km}$ entre si, pertencentes ao município de S.J.Barreiro (Figura 1). A primeira área está localizada na Fazenda Atibaia (22 $38^{\prime} 34^{\prime \prime}$ S e $\left.44^{\circ} 39^{\prime} 24^{\prime \prime} \mathrm{W}\right)$, a $504 \mathrm{~m}$ de altitude, que abriga inúmeros remanescentes de Floresta Estacional Semidecidual, doravante tratada como FE. Está inserida na região de "mares de morros", pertencendo à Bacia Hidrográfica do rio Paraíba do Sul. O clima da região é quente e úmido, com inverno seco ou Cwa de Koppen, sendo a temperatura média anual de $20^{\circ} \mathrm{C}$ (temperatura do mês mais frio inferior a $18^{\circ} \mathrm{C}$ e a do mês mais quente superior a $22{ }^{\circ} \mathrm{C}$ ), tendo uma precipitação média anual

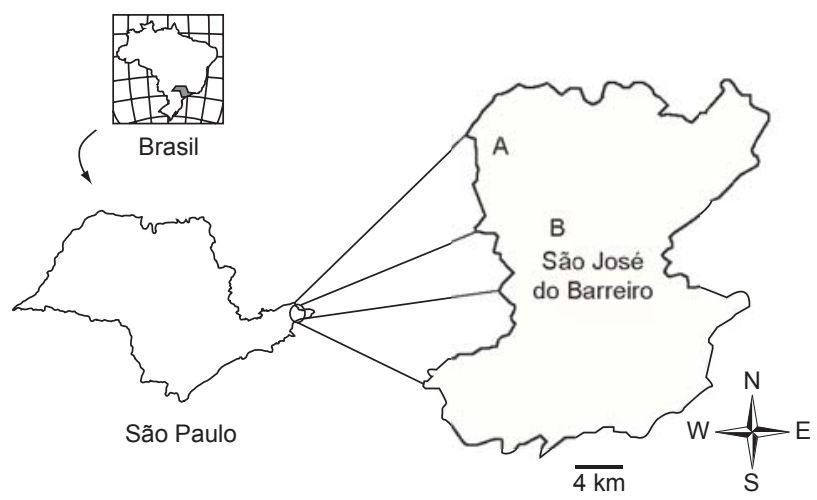

Figura 1. Mapa mostrando a localização das áreas de estudo em São José do Barreiro, estado de São Paulo, Brasil. A) Floresta Estacional Semidecidual; e B) Floresta Ombrófila Densa.

Figure 1. Maps showing of the study areas in São José do Barreiro municipality, State of São Paulo, Brazil. A) Seasonal Semideciduous Forest; and B) Tropical Rainforest. 
de $1.500 \mathrm{~mm}$ (Vasconcelos 1992). A segunda área está concentrada na propriedade da Pousada Recanto da Floresta ( $22^{\circ} 43^{\prime} 08.4^{\prime \prime} \mathrm{S}$ e $044^{\circ} 36^{\prime} 51.0^{\prime \prime} \mathrm{W}$ ), entre as cotas 1.510 e $1.950 \mathrm{~m}$ de altitude, sendo constituída por Campos de Altitude, fisionomia não amostrada neste estudo, e principalmente por remanescentes de Floresta Ombrófila Densa, e doravante será tratada como área FO. Está situada na região da Serra da Bocaina, e inserida na Bacia Hidrográfica do Rio Mambucaba. Esta região do planalto da Bocaina apresenta um clima tropical de altitude com característica superúmida, com temperatura média anual inferior a $17{ }^{\circ} \mathrm{C}$, tendo durante os meses de inverno, principalmente junho e julho, temperaturas inferiores a $0{ }^{\circ} \mathrm{C}$, o que ocasiona a formação de geadas. A precipitação média anual é de 2.100 mm (MMA/IBAMA 2004). As duas áreas deste estudo situam-se na Zona de amortecimento do Parque Nacional da Serra da Bocaina (SP/RJ) e se constituem um mosaico de relictos florestais primários, formações florestais secundárias em diferentes estádios de regeneração (SP: SMA/IF 2005), campos de altitude antropizados e sobretudo pastagens (MMA/IBAMA 2004).

\section{Amostragem e análise dos dados}

Foram realizados 18 períodos de amostragem na área de FE, entre os meses de abril de 2004 e dezembro de 2006 (primeira quinzena de abril/2004; segunda quinzena de abril/2004 a setembro/2004; novembro/2004 a janeiro/2005; março/2005; maio/2005; julho/2005; dezembro/2005; fevereiro/2006; junho/2006; agosto/2006 e dezembro/2006), e oito períodos de amostragem na área de FO, entre maio de 2005 e dezembro de 2006 (maio/2005; agosto/2005; primeira quinzena de dezembro/2005; segunda quinzena de dezembro/2005; fevereiro/2006; junho/2006; agosto/2006 e dezembro/2006). As amostragens tiveram duração de dois a quatro dias e a metodologia utilizada foi a de procura auditiva e encontros ocasionais, realizada no período diurno e noturno, sem limite de tempo. A nomenclatura considerada neste trabalho foi a de Faivovich et al. (2005), Frost et al. (2006), Grant et al. (2006) e Chaparro et al. (2007). Espécimestestemunho encontram-se depositados na Coleção de Vertebrados Dr. Jorge Jim (CVJJ), do Departamento de Zoologia da Universidade Estadual Paulista, Campus de Botucatu, SP e na Coleção "Célio F. B. Haddad" (CFBH), do Departamento de Zoologia da Universidade Estadual Paulista, Campus de Rio Claro, SP.

Para cada uma das áreas, foi calculada a constância de ocorrência das espécies (C) modificada de Dajoz (1972) para melhor interpretação dos resultados. De acordo com os valores de $\mathrm{C}$, foram definidas as seguintes categorias: a) espécies comuns, presentes em mais de $50 \%$ da amostragem; b) relativamente comuns, entre $25 \%$ e $50 \%$ e c) esporádicas em menos de $25 \%$ da amostragem. Foram estabelecidas comparações entre a taxocenose de anuros das áreas estudadas com as taxocenoses de outras seis localidades do Estado de São Paulo: Estação Ecológica de Bananal (E.E.Bananal), na região do Vale do Paraíba (Zaher et al. 2005); Estação Biológica de Boracéia (E.B.Boracéia) (Heyer et al. 1990); Serra do Japi, na região de Jundiaí (Haddad \& Sazima 1992); Nova Itapirema, região Noroeste de São Paulo (Vasconcelos \& Rossa-Feres 2005); Parque Estadual de Intervales (P.E. Intervales), na região de Capão Bonito (Bertoluci 2001) e Estação Ecológica Juréia-Itatins (E.E.Juréia-Itatins), na região de Peruíbe (Pombal Jr. \& Gordo 2004). A comparação foi efetuada por análise de agrupamento pelo método de média não ponderada, aplicada na matriz do coeficiente de Jaccard (Krebs 1999) para os dados de presença e ausência de cada espécie nas diferentes localidades, ambas realizadas no programa Cluster, versão 1.3 (programa desenvolvido por Dr. George Shepherd, do Departamento de Botânica, IB, Unicamp). A comparação da composição de taxocenoses de diferentes localidades pode ser deturpada e até irreal em decorrência de três aspectos citados por Pombal Jr. (1995): inventários com diferentes esforços amostrais, diferenças no tamanho e características das áreas e diferenças nos conceitos taxonômicos entre os autores. A fim de minimizar tais problemas, a análise de similaridade levou em consideração apenas as espécies determinadas e aquelas citadas como confer (cf.) ou grupo (gr.) (e.g. Ischnocnema gr. lactea = I. lactea) e diferenças no tamanho das áreas e no esforço amostral dos inventários foram abordadas na discussão.

\section{Resultados}

Foram registradas 35 espécies pertencentes a 14 gêneros de nove famílias de anfíbios anuros (Tabela 1; Figura 2), sendo 19 espécies na FE e 19 na FO. As famílias com menor número de representantes na região foram Amphignatodontidae, Centrolenidae, Cycloramphidae e Hylodidae, cada uma com uma espécie. As duas áreas apresentaram composição distinta de espécies de anfíbios anuros (Tabela 1), tendo apenas três espécies em comum, Dendropsophus minutus, Hypsiboas cf. polytaenius e Rhinella icterica. A curva do coletor considerando conjuntamente as duas áreas amostradas não apresentou tendência a atingir a assíntota (Figura 3).

Com relação aos tipos de hábitats ocupados pelas espécies, encontramos, como esperado, dois padrões distintos associados às condições climáticas locais e respectivos tipos florestais (Tabela 1). A anurofauna da região da $\mathrm{FO}$ (presente estudo) apresentou padrão típico de áreas de florestas tropicais úmidas: algumas espécies $(15,8 \%)$ apresentam reprodução terrestre, utilizando refúgios na serapilheira para deposição de ovos, outras $(10,5 \%)$ apresentam reprodução associada a bromélias, e a maioria $(73,7 \%)$ apresenta reprodução associada a ambientes aquáticos. Em relação à área da FE, que apresenta uma estação seca e outra chuvosa, não foram registradas espécies com sítio de vocalização associado a bromélias, sendo encontrada apenas uma espécie (5\%) com reprodução terrestre e a grande maioria com reprodução associada a ambientes aquáticos $(95 \%)$.

O índice de constância para as espécies amostradas na região da FO (Figura 4) apresentou três espécies comuns (Aplastodiscus leucopygius, Hypsiboas cf. polytaenius e Rhinella icterica), 13 espécies relativamente comuns (Aplastodiscus perviridis, Bokermannohyla circumdata, Dendropsophus microps, Ischnocnema guentheri, Ischnocnema gr. lactea, I. parva, Flectonotus sp., Leptodactylus sp., Physalaemus olfersii, Scinax fuscomarginatus, S. hayii, S. perpusillus e $S$. squalirostris), e três esporádicas (D. minutus, Hylodes sp. e Proceratophrys boiei). Para a área da FE o índice de constância (Figura 5) indicou seis espécies comuns (D. minutus, Hypsiboas faber, Leptodactylus ocellatus, Rhinella icterica, $R$. ornata e $S$. fuscovarius), cinco espécies relativamente comuns (D. elegans, Hypsiboas pardalis, Leptodactylus fuscus, Phyllomedusa burmeisteri e Physalaemus cuvieri), e oito esporádicas (Eleutherodactylus binotatus, Hyalinobatrachium eurygnathum, Hypsiboas albopunctatus, $H$. cf. polytaenius, H. semilineatus, Leptodactylus labyrinthicus, $S$. humilis e $S$. similis).

A análise de similaridade considerando a anurofauna de seis áreas já estudadas (E.B.Boracéia, E.E.Bananal, P.E.Intervales, Serra do Japi, Nova Itapirema e E.E.Juréia-Itatins) e das áreas FE e FO consideradas separadamente, está apresentada na Figura 6a. A análise de agrupamento separou inicialmente Nova Itapirema das demais localidades. E.E.Bananal, FO, Serra do Japi, E.B.Boracéia, P.E.Intervales e FE formam um grupo do qual se separa a E.E.Juréia-Itatins. Em seguida, a área FE separa-se do grupo restante (E.E.Bananal, FO, Serra do Japi, E.B.Boracéia e P.E.Intervales). As cinco áreas restantes formam dois grupos: um formado pela E.B. Boracéia e P.E.Intervales e outro formado pela E.E.Bananal, FO e Serra do Japi. Dentro deste grupo, E.E.Bananal e FO estão unidos.

Considerando as áreas FE e FO em conjunto, tratadas como S.J.Barreiro (Figura 6b), a análise de agrupamento separou inicial- 
Tabela 1. Espécies de anuros registradas neste estudo em São José do Barreiro, estado de São Paulo, Brasil. Floresta Ombrófila Densa (FO); Floresta Estacional Semidecidual (FE). (*) Espécies acrescentadas ao inventário preliminar de Bananal, SP (Zaher et al. 2005). B: sobre bromélias; BA: brejos em áreas abertas; L: lagoas; PA: poça temporária em área aberta; PM: poça temporária na mata; R: riachos na mata; RL: riachos lentos de pastagens; S: serapilheira; SD: sem dados; V: várzea.

Table 1. Amphibian anurans registered in the present study in São José do Barreiro, São Paulo state, Brazil. Tropical Rainforest (FO); Seasonal Semideciduous Forest (FE). (*) Species added to preliminar survey in the Bananal reserve (Zaher et al. 2005). B:on bromeliads; BA: swamps in open areas; L: permanent ponds; PA: temporary ponds in open areas; PM: temporary ponds in the middle of forest; R: forest streams; RL: slow pasture stream; S: leaf litter; SD: no data; V: low and flat land alongside a watercourse.

\begin{tabular}{|c|c|c|c|}
\hline Espécie & Nome popular & Ambiente reprodutivo & Formação florestal \\
\hline \multicolumn{4}{|l|}{ Amphignatodontidae } \\
\hline *Flectonotus sp. & perereca-da-bromélia & B & FO \\
\hline \multicolumn{4}{|l|}{ Brachycephalidae } \\
\hline Eleutherodactylus binotatus & rã-da-mata & S & $\mathrm{FE}$ \\
\hline Ischnocnema guentheri & rã-da-mata & S & FO \\
\hline * Ischnocnema gr. lactea & rã-da-mata & $S$ & FO \\
\hline Ischnocnema parva & rãzinha-da-mata & $S$ & $\mathrm{FO}$ \\
\hline \multicolumn{4}{|l|}{ Bufonidae } \\
\hline Rhinella icterica & sapo cururu, cururuzão & $L, R L$ & $F E / F O$ \\
\hline *Rhinella ornata & cururuzinho, sapo-da-mata & $L, R L, R$ & $F E$ \\
\hline \multicolumn{4}{|l|}{ Centrolenidae } \\
\hline *Hyalinobatrachium eurygnathum & rã-de-vidro & SD & $\mathrm{FE}$ \\
\hline \multicolumn{4}{|l|}{ Cycloramphidae } \\
\hline Proceratophrys boiei & sapo-de-chifre & SD & FO \\
\hline \multicolumn{4}{|l|}{ Hylidae } \\
\hline Aplastodiscus leucopygius & perereca-flautinha & $\mathrm{R}$ & FO \\
\hline *Aplastodiscus perviridis & perereca-verde & $\mathrm{BA}, \mathrm{R}$ & FO \\
\hline Bokermannohyla circumdata & perereca-castanhola & $\mathrm{R}$ & FO \\
\hline Dendropsophus elegans & perereca-de-moldura & $\mathrm{L}, \mathrm{PA}$ & FE \\
\hline Dendropsophus microps & pererequinha-do-brejo & PA, PM & FO \\
\hline Dendropsophus minutus & perereca-de-ampulheta & $\mathrm{L}, \mathrm{PA}$ & $\mathrm{FE} / \mathrm{FO}$ \\
\hline *Hypsiboas albopunctatus & perereca-marrom & $\mathrm{L}, \mathrm{RL}$ & FE \\
\hline Hypsiboas faber & sapo-ferreiro & $\mathrm{L}, \mathrm{PA}$ & $\mathrm{FE}$ \\
\hline Hypsiboas pardalis & perereca-líquen & $\mathrm{L}, \mathrm{RL}$ & $\mathrm{FE}$ \\
\hline Hypsiboas cf. polytaenius & perereca-de-pijama & $\mathrm{RL}, \mathrm{BA}$ & $\mathrm{FO} / \mathrm{FE}$ \\
\hline *Hypsiboas semilineatus & perereca-cacarejo & $\mathrm{L}$ & $\mathrm{FE}$ \\
\hline *Phyllomedusa burmeisteri & perereca-verde-e-amarela & $\mathrm{L}, \mathrm{PA}$ & FE \\
\hline *Scinax fuscomarginatus & perereca-do-brejo & BA & FO \\
\hline *Scinax fuscovarius & perereca-de-banheiro & BA & $\mathrm{FE}$ \\
\hline Scinax hayii & perereca-raspa-cuia & BA, PA & $\mathrm{FO}$ \\
\hline *Scinax humilis & perereca-risonha & $\mathrm{PM}$ & $\mathrm{FE}$ \\
\hline Scinax perpusillus & pererequinha-das-bromélias & $\mathrm{B}$ & FO \\
\hline$*$ Scinax similis & perereca-da-mata & SD & $\mathrm{FE}$ \\
\hline *Scinax squalirostris & perereca-bicuda & BA, PA, PM & $\mathrm{FO}$ \\
\hline \multicolumn{4}{|l|}{ Hylodidae } \\
\hline Hylodes sp. & rã-do-riacho & $\mathrm{R}$ & FO \\
\hline \multicolumn{4}{|l|}{ Leiuperidae } \\
\hline *Physalaemus cuvieri & rã-cachorro & L, BA, V & $\mathrm{FE}$ \\
\hline Physalaemus olfersii & rã-gemedeira, rã-chorona & BA & FO \\
\hline \multicolumn{4}{|l|}{ Leptodactylidae } \\
\hline *Leptodactylus fuscus & rã-assoviadora & $\mathrm{V}$ & $\mathrm{FE}$ \\
\hline *Leptodactylus labyrinthicus & rã-pimenta & SD & $\mathrm{FE}$ \\
\hline *Leptodactylus ocellatus & rã-manteiga & $\mathrm{L}$ & $\mathrm{FE}$ \\
\hline Leptodactylus sp. & rã-da-várzea & $\mathrm{V}$ & FO \\
\hline
\end{tabular}



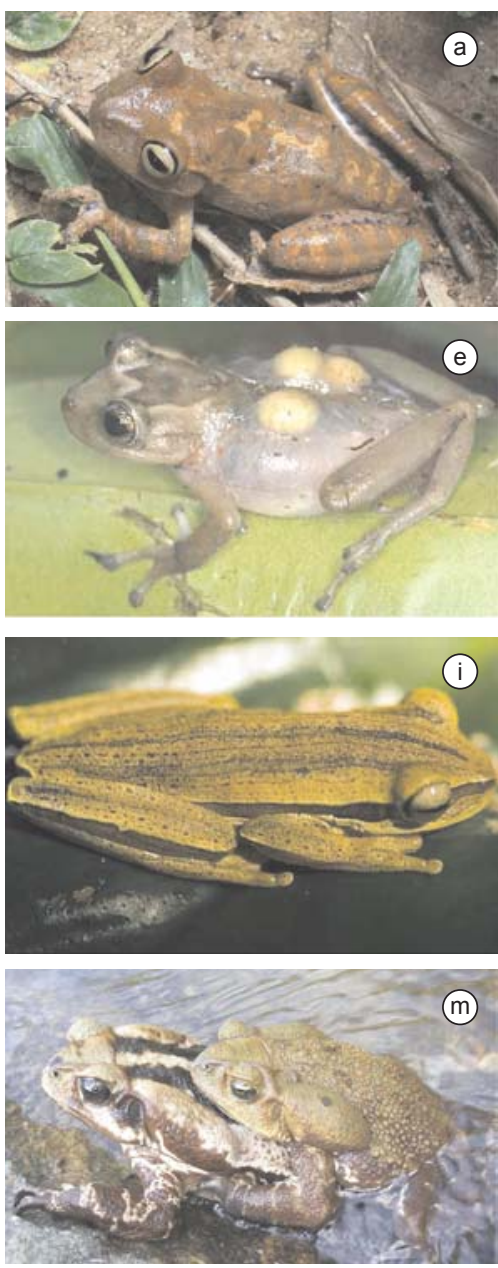
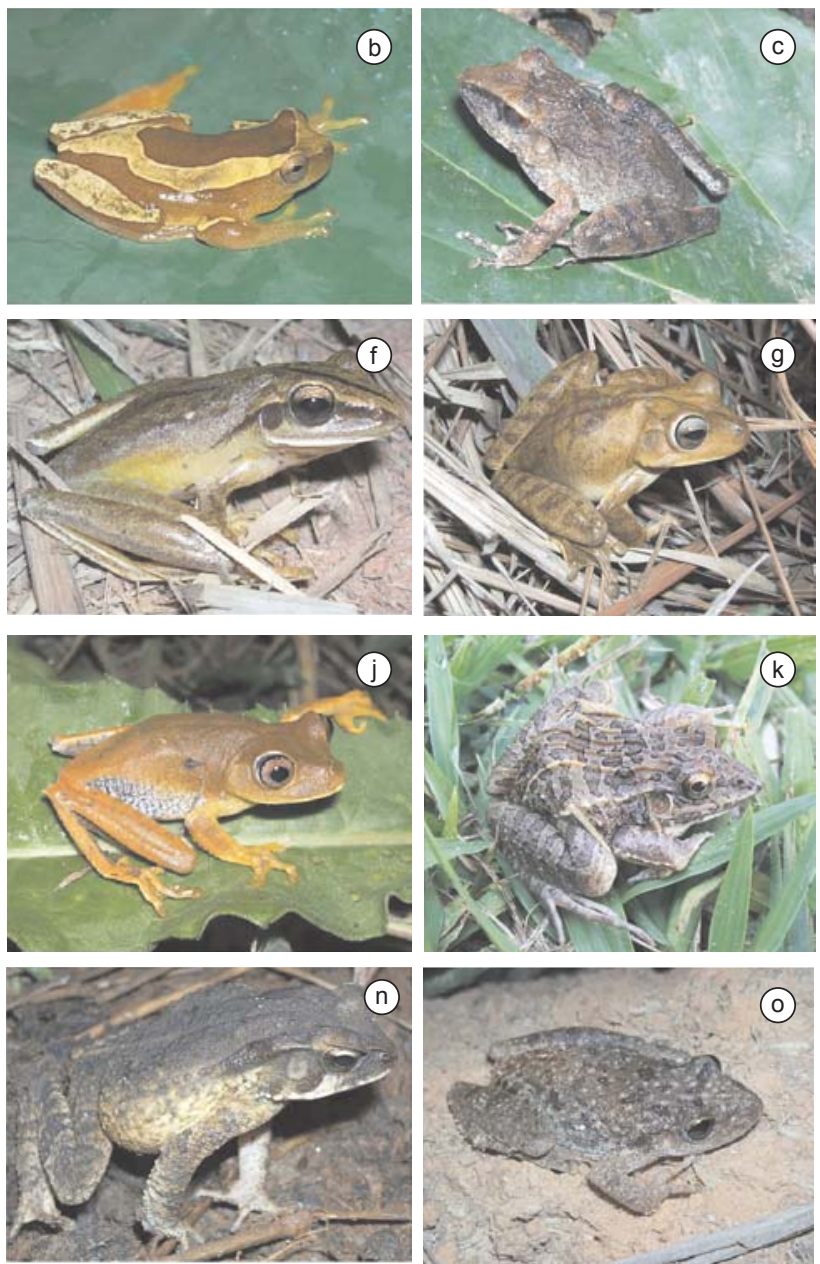

(0)

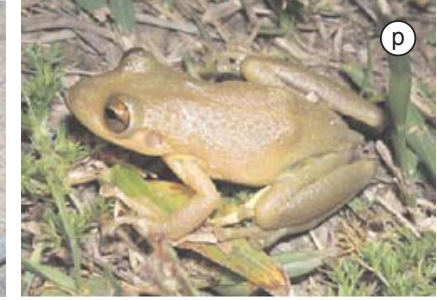

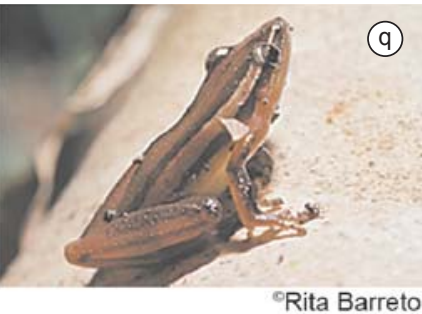

Figura 2. Espécies de anuros encontradas nas áreas de estudo do município de São José do Barreiro, SP. a) Bokermannohyla circumdata, b) Dendropsophus elegans, c) Eleutherodactylus binotatus, d) I. gr. lactea, e) Flectonotus sp., f) Hypsiboas albopunctatus, g) H. faber, h) H. pardalis, i) H. cf. polytaenius, j) H. semilineatus, k) Leptodactylus fuscus, l) L. ocellatus, m) Rhinella icterica, n) R. ornata, o) Scinax fuscovarius, p) S. hayii e q) S. squalirostris.

Figure 2. Anurans species found in study áreas in São José do Barreiro municipality, SP. a) Bokermannohyla circumdata, b) Dendropsophus elegans, c) Eleutherodactylus binotatus, d) I. gr. lactea e) Flectonotus sp., f) Hypsiboas albopunctatus, g) H. faber, h) H. pardalis, i) H. cf. polytaenius, j) H. semilineatus, k) Leptodactylus fuscus, 1) L. ocellatus, m) Rhinella icterica, n) R. ornata, o) Scinax fuscovarius, p) S. hayii and q) S. squalirostris.

mente Nova Itapirema das demais localidades. E.E.Bananal, Serra do Japi, S.J.Barreiro, E.B.Boracéia, P.E.Intervales e E.E.Juréia-Itatins formam um grupo do qual se separa a E.E.Juréia-Itatins. As cinco áreas restantes formam dois grupos: um formado pela E.B.Boracéia e P.E.Intervales e outro formado pela E.E.Bananal, Serra do Japi e S.J.Barreiro. Dentro deste grupo, Serra do Japi e S.J.Barreiro estão unidos, apesar da menor distância entre S.J.Barreiro e a E.E.Bananal.

\section{Discussão}

A família Hylidae apresentou o maior número de espécies nas duas formações florestais estudadas, o que está de acordo com o padrão encontrado na região Neotropical (Heyer et al. 1990, Duellman 1988), e em diversas áreas do Domínio da Floresta Atlântica (Heyer et al. 1990, Haddad \& Sazima 1992, Pombal Jr. \& Gordo 2004, Vasconcelos \& Rossa-Feres 2005).

Nenhuma das espécies registradas em S.J. do Barreiro encontrase na lista nacional de espécies ameaçadas do IBAMA (2003) ou nas listas de espécies ameaçadas de São Paulo (São Paulo 1998), Minas Gerais (Machado et al. 1998) e Rio de Janeiro (Bergallo et al. 2000).

Os índices de constância para as espécies amostradas na região da FE (Figura 5) destacam a presença de espécies ecologicamente generalistas de áreas abertas, como: Dendropsophus elegans D. minutus, 


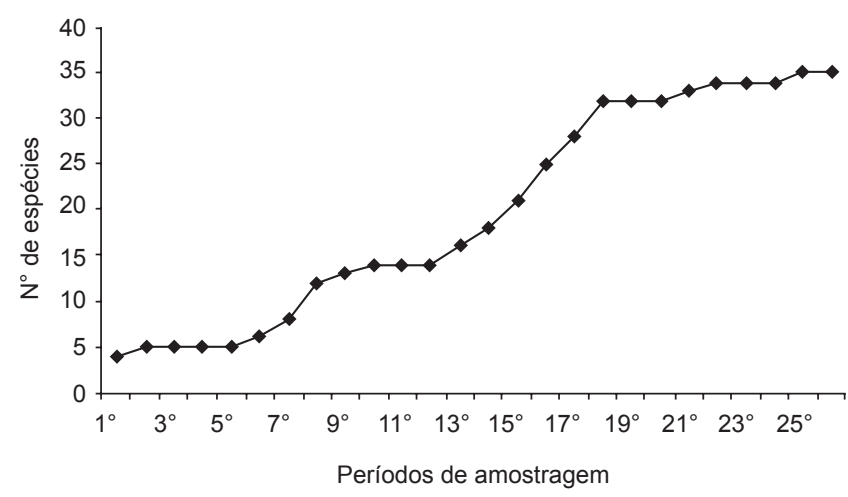

Figura 3. Curva de acumulação de espécies para os 26 períodos de amostragem realizados no município de São José do Barreiro, São Paulo, Brasil.

Figure 3. Accumulation curve of species for 26 samples carrying out in São José do Barreiro municipality, São Paulo, Brazil.

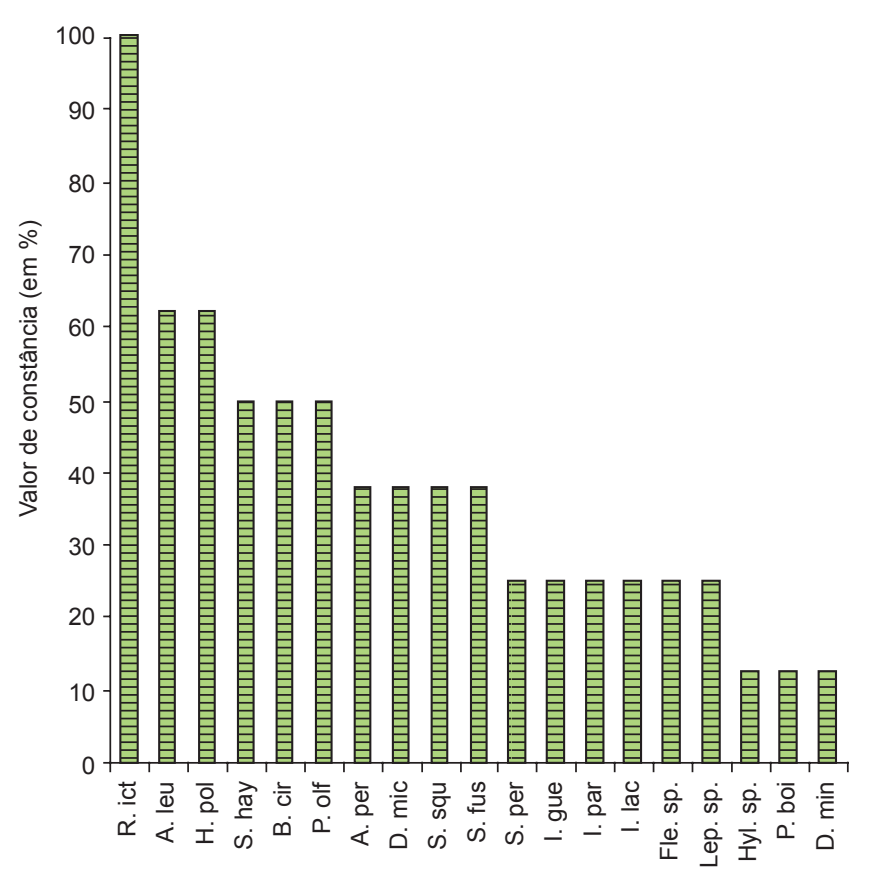

Figura 4. Constância de ocorrência das espécies amostradas na Pousada Recanto da Floresta, Serra da Bocaina, São José do Barreiro (SP). Rhinella icterica (R. ict); Aplastodiscus leucopygius (A. leu); Hypsiboas cf. polytaenius (H. pol); Scinax hayii (S. hay); Bokermannohyla circumdata (B. cir); Physalaemus olfersii (P.olf); Aplastodiscus perviridis (A. per); Dendropsophus microps (D. mic); Scinax squalirostris (S. squ); Scinax fuscomarginatus (S. fus); Scinax perpusillus (S. per); Ischnocnema guentheri (I. gue); Ischnocnema parva (I. par); Ischnocnema gr. lactea (I. lac); Flectonotus sp. (Fle. sp.); Leptodactylus sp. (Lep. sp.); Hylodes sp. (Hyl. sp.); Proceratophrys boiei (P. boi); Dendropsophus minutus (D. min).

Figure 4. Occurrence constancy of species registered in the Pousada Recanto da Floresta, Serra da Bocaina, São José do Barreiro (SP). Rhinella icterica (R. ict); Aplastodiscus leucopygius (A. leu); Hypsiboas cf. polytaenius (H. pol); Scinax hayii (S. hay); Bokermannohyla circumdata (B. cir); Physalaemus olfersii (P. olf); Aplastodiscus perviridis (A. per); Dendropsophus microps (D. mic); Scinax squalirostris (S. squ); Scinax fuscomarginatus (S.f us); Scinax perpusillus (S. per); Ischnocnema guentheri (I. gue); Ischnocnema parva (I. par); Ischnocnema gr. lactea (I. lac); Flectonotus sp. (Fle. sp.); Leptodactylus sp. (Lep. sp.); Hylodes sp. (Hyl. sp.); Proceratophrys boiei (P. boi); Dendropsophus minutus (D. min).

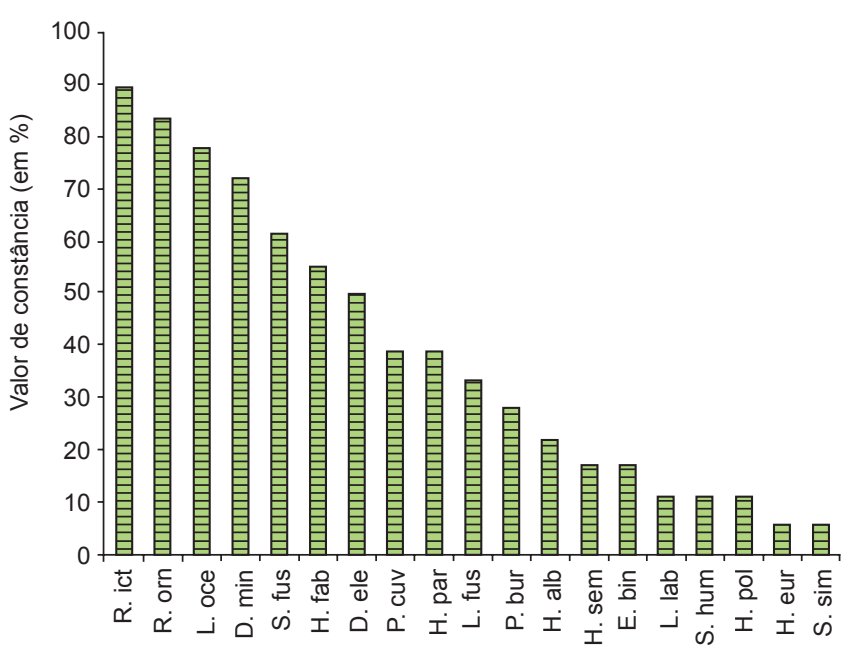

Figura 5. Constância de ocorrência das espécies amostradas na região da Fazenda Atibaia, São José do Barreiro, SP. Rhinella icterica (R. ict); Rhinella ornata (R. orn); Leptodactylus ocellatus (L. oce); Dendropsophus minutus (D. min); Scinax fuscovarius (S. fus); Hypsiboas faber (H. fab); Dendropsophus elegans (D. ele); Physalaemus cuvieri (P. cuv); Hypsiboas pardalis (H. par); Leptodactylus fuscus (L. fus); Phyllomedusa burmeisteri (P. bur); Hypsiboas albopunctatus (H. alb); Hypsiboas semilineatus (H. sem); Eleutherodactylus binotatus (E. bin); Leptodactylus labyrinthicus (L. lab); Scinax humilis (S. hum); Hypsiboas cf. polytaenius (H. pol); Hyalinobatrachium eurygnathum (H. eur); Scinax similis (S. sim).

Figure 5. Occurrence constancy of species registered in the Atibaia farm, São José do Barreiro, SP. Rhinella icterica (R. ict); Rhinella ornata (R. orn); Leptodactylus ocellatus (L. oce); Dendropsophus minutus (D. min); Scinax fuscovarius (S. fus); Hypsiboas faber (H. fab); Dendropsophus elegans (D. ele); Physalaemus cuvieri (P. cuv); Hypsiboas pardalis (H. par); Leptodactylus fuscus (L. fus); Phyllomedusa burmeisteri (P. bur); Hypsiboas albopunctatus (H. alb); Hypsiboas semilineatus (H. sem); Eleutherodactylus binotatus (E. bin); Leptodactylus labyrinthicus (L. lab); Scinax humilis (S. hum); Hypsiboas cf. polytaenius (H. pol); Hyalinobatrachium eurygnathum (H. eur); Scinax similis (S. sim).

Hypsiboas faber, H. pardalis, Leptodactylus fuscus, L. ocellatus, Physalaemus cuvieri, Rhinella icterica, $R$. ornata e Scinax fuscovarius (Heyer et al. 1990, Haddad \& Sazima 1992, Haddad 1998, Izecksohn \& Carvalho-e-Silva 2001, Brasileiro et al. 2005, Conte \& Rossa-Feres 2006). Por outro lado, a região da FO (Figura 4) apresenta diversas espécies associadas a hábitats florestados como: Aplastodiscus leucopygius, Bokermannohyla circumdata, Ischnocnema guentheri, I. parva, Flectonotus sp. e Physalaemus olfersii (Heyer et al. 1990, Izecksohn \& Carvalho-e-Silva 2001, Haddad \& Prado 2005, Moraes et al. 2007). Entre os fatores que podem explicar essa diferença estão: maior cobertura florestal existente na região da FO, melhor estado de conservação (MMA/IBAMA 2004) e maior umidade ao longo do ano (Haddad \& Prado 2005), quando comparada à área da FE, que apresenta estação seca pronunciada (Martins et al. 2002, MMA/IBAMA 2004). Aspectos como a capacidade das espécies de colonizar áreas alteradas, tolerâncias fisiológicas distintas e a necessidade de microambientes específicos para a reprodução são possíveis fatores responsáveis pelos distintos padrões de frequiência das espécies nas duas áreas estudadas (Jim 1980, Haddad \& Prado 2005, Moraes et al. 2007).

O método de procura ativa permitiu a amostragem dos inúmeros ambientes que compõem as áreas estudadas, porém mostrou menor eficiência na amostragem de espécies tipicamente associadas à 
serapilheira. Tal aspecto pode ser verificado se considerarmos a ausência de algumas espécies registradas na E.E.Bananal $\left(22^{\circ} 68^{\prime} \mathrm{S}\right.$ e $44^{\circ} 32^{\prime} \mathrm{W}$ ) por Zaher et al. (2005), que utilizaram armadilhas de interceptação e queda para o levantamento, como as dos gêneros Brachycephalus, Chiasmocleis, Cycloramphus, Holoaden, Myersiella e Paratelmatobius. Ainda assim, o presente estudo acrescenta 17 espécies de anuros (Tabela 1) ao inventário preliminar realizado por Zaher et al. (2005), na E.E.Bananal, que dista cerca de $24 \mathrm{~km}$ da área de FO amostrada neste estudo. Dessas 17 espécies, Aplastodiscus perviridis, Ischnocnema gr. lactea, Flectonotus sp., Scinax fuscomarginatus e $S$. squalirostris foram coletadas na área de FO, mesma tipologia florestal da área estudada na E.E.Bananal, as 12 restantes foram encontradas nas área de FE do município de
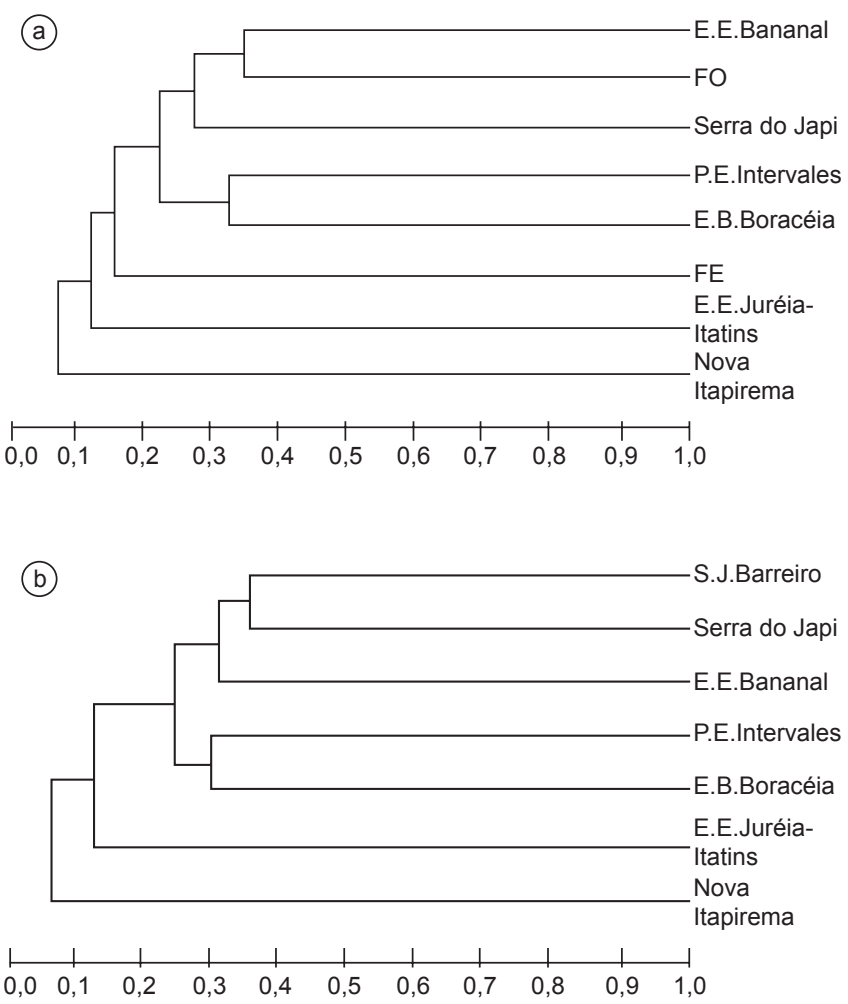

Figura 6. Dendrograma da análise de agrupamento resultante da presença e ausência das espécies de anfíbios anuros de sete taxocenoses da Floresta Atlântica do Estado de São Paulo, Brasil. a) Floresta Ombrófila Densa (FO); Floresta Estacional Semidecidual (FE); Estação Ecológica de Bananal (E.E.Bananal); Estação Biológica de Boracéia (E.B.Boracéia); Serra do Japi; Nova Itapirema; Parque Estadual de Intervales (P.E.Intervales) e Estação Ecológica Juréia-Itatins (E.E.Juréia-Itatins). e b) S.J.Barreiro (FE+FO); Estação Ecológica de Bananal (E.E.Bananal); Estação Biológica de Boracéia (E.B.Boracéia); Serra do Japi; Nova Itapirema; Parque Estadual de Intervales (P.E.Intervales) e Estação Ecológica Juréia-Itatins (E.E.Juréia-Itatins).

Figure 6. Cluster analysis dendrogram of anurans presence and absence in seven assemblages from Atlantic Forest areas of State of São Paulo, Brazil. a) Tropical Rainforest (FO); Seasonal Semideciduous Forest (FE); Estação Ecológica de Bananal (E.E.Bananal); Estação Biológica de Boracéia (E.B.Boracéia); Serra do Japi; Nova Itapirema; Parque Estadual de Intervales (P.E.Intervales) and Estação Ecológica Juréia-Itatins (E.E.Juréia-Itatins). and b) S.J.Barreiro (FE+FO); Estação Ecológica de Bananal (E.E.Bananal); Estação Biológica de Boracéia (E.B.Boracéia); Serra do Japi; Nova Itapirema; Parque Estadual de Intervales (P.E.Intervales) and Estação Ecológica Juréia-Itatins (E.E.Juréia-Itatins).
S.J.Barreiro. Considerando ainda a formação florestal em que cada espécie foi coletada, nestes dois estudos, a Floresta Ombrófila Densa comportou maior riqueza (35 espécies) que a Floresta Estacional Semidecidual (19 espécies).

Ao compararmos diversas listas de espécies publicadas para localidades do Estado de São Paulo, a despeito das diferenças nos períodos e esforço de amostragem e no número de levantamentos efetuados nas diferentes regiões, o inventário realizado neste estudo apresenta uma riqueza de espécies elevada, sendo superior a de algumas localidades bem preservadas de Mata Atlântica do Estado de São Paulo (Tabela 2), como a Serra do Japi (31 espécies; Haddad \& Sazima 1992, Ribeiro et al. 2005), E.E.Bananal (30 espécies; Zaher et al. 2005) e a E.E.Juréia-Itatins (26 espécies; Pombal \& Gordo 2004).

O padrão de similaridade obtido entre as taxocenoses de anuros da E.B.Boracéia, E.E.Bananal, P.E.Intervales, Serra do Japi, Nova Itapirema, E.E.Juréia-Itatins, FE e FO (Figura 6a) parece coerente com as fisionomias vegetais das áreas, caracterizadas por Veloso et al. 1991. A similaridade na composição da anurofauna da E.E.Bananal, FO (presente estudo) e Serra do Japi pode ser explicada pelo fato de estas localidades estarem situadas nos domínios da Floresta Ombrófila Densa (FO e E.E. Bananal) ou na transição desta para a Floresta Estacional Semidecidual (Serra do Japi) (Ribeiro et al. 2005, SP: SMA/IF 2005). Apesar do grau variado de interferência antrópica nessas localidades, essas regiões encontram-se no Planalto Atlântico, em áreas situadas nos reversos da Serra do Mar (FO e E.E.Bananal) e na Serra do Japi, o que resulta em menores índices pluviométricos e distribuição mais irregular da precipitação (Morellato et al. 1989, Leitão Filho 1992, MMA/IBAMA 2004). Dentro deste grupo, a menor distância entre FO e E.E.Bananal, cerca de 24 km, também deve ter contribuído para a maior semelhança das áreas. Por outro lado, a similaridade entre E.B.Boracéia e o P.E.Intervales pode estar relacionada à localização de ambas na Província Costeira, na Subzona da Serrania Costeira, nas escarpas da Serra voltadas para o Mar, e pelo fato de serem recobertos por remanescentes de Florestas Ombrófilas Densas. A E.B.Boracéia e o P.E.Intervales apresentam elevados valores de precipitação, $3.058 \mathrm{~mm}$ e $4.000 \mathrm{~mm}$, respectivamente, que são bem distribuídos ao longo do ano, o que resulta em ausência de

Tabela 2. Número de espécies de anuros registradas em sete localidades de Mata Atlântica do estado de São Paulo. Estação Ecológica (E.E.); Parque Estadual (P.E.); Estação Biológica (E.B.). Floresta Ombrófila Densa (FO); Floresta Estacional Semidecidual (FE).

Table 2. Number of anuran species recorded for seven localities within the Atlantic Forest, São Paulo state, Brazil. Ecological Station (E.E.); State Park (P.E.); Biological Station (E.B.). Atlantic Rainforest (FO); Seasonal Semideciduous Forest (FE).

\begin{tabular}{|c|c|c|c|}
\hline Localidades & $\begin{array}{l}\text { Formação } \\
\text { Florestal }\end{array}$ & $\mathrm{N}^{\circ}$ espécies & Referências \\
\hline E.E. Juréia & FO & 26 & $\begin{array}{l}\text { Pombal \& } \\
\text { Gordo (2004) }\end{array}$ \\
\hline P.E. Intervales & $\mathrm{FO}$ & 48 & Bertoluci (2001) \\
\hline Nova Itapirema & $\mathrm{FE}$ & 27 & $\begin{array}{l}\text { Vasconcelos \& } \\
\text { Rossa-Feres (2005) }\end{array}$ \\
\hline Serra do Japi & FE & 31 & $\begin{array}{l}\text { Haddad \& Sazima } \\
(1992), \text { Ribeiro et al. } \\
(2005)\end{array}$ \\
\hline E.B. Boracéia & $\mathrm{FO}$ & 65 & Heyer et al. (1990) \\
\hline E.E. Bananal & $\mathrm{FO}$ & 30 & Zaher et al. (2005) \\
\hline $\begin{array}{l}\text { São José } \\
\text { do Barreiro }\end{array}$ & $\mathrm{FE}+\mathrm{FO}$ & 35 & Presente estudo \\
\hline
\end{tabular}


período seco (Heyer et al. 1990, Morellato et al. 2000). A região FE apresentou baixa similaridade com as duas localidades acima, provavelmente por apresentar clima sazonal (MMA/IBAMA 2004), com verões quentes e úmidos (Leitão Filho 1982) e invernos secos e frios, quando cerca de 50 a $70 \%$ de suas espécies perdem as folhas (Morellato et al. 1989, Veloso et al. 1991, Morellato \& Leitão Filho 1992). Diversos estudos têm mostrado que a atividade reprodutiva dos anuros é fortemente influenciada pelo regime pluviométrico, o que reflete na composição da taxocenose das diferentes regiões (Duellman 1978, Rossa-Feres \& Jim 1994, Vasconcelos \& Rossa-Feres 2005).

A E.E.Juréia-Itatins está situada na Província Costeira, na subzona da Baixada Litorânea. Porém, a E.E.Juréia-Itatins diverge da E.B.Boracéia e do P.E.Intervales, que também se situam na Província Costeira, por apresentar um predomínio de Florestas de Restinga, que foi a principal fisionomia amostrada no estudo de Pombal Jr. \& Gordo (2004), o que deve ter refletido na menor similaridade com as demais localidades. De acordo com o registrado por Pombal Jr. \& Gordo (2004), existe maior similaridade da anurofauna da E.E.Juréia-Itatins com outras áreas da baixada litorânea, em detrimento de áreas de maior altitude localizadas mais próximas da Serra do Mar ou do Planalto Atlântico, que apresentam condições topográficas e climáticas distintas da Estação E.E.Juréia-Itatins. Por fim, a menor similaridade da taxocenose de anuros de Nova Itapirema com as demais regiões decorre, provavelmente, do fato de a região se situar em área de transição entre o Cerrado e a Floresta Estacional Semidecidual, que favorece a ocorrência de espécies de anuros de áreas abertas (Brasileiro et al. 2005, Vasconcelos \& Rossa-Feres 2006).

$\mathrm{Na}$ análise de agrupamento, considerando em conjunto as duas áreas estudadas (Figura 6b), a E.E.Bananal, Serra do Japi e S.J.Barreiro estão associados em um grupo com maior similaridade que as demais localidades. Dentro deste grupo, Serra do Japi e S.J.Barreiro tem maior similaridade, apesar da menor distância, de cerca de 24 km entre elas, o que sugere menor influência da distância geográfica na similaridade. Pombal Jr. \& Gordo (2004) já haviam salientado que a distância não é o único fator a determinar a semelhança entre taxocenoses de anuros e ressaltaram a importância de aspectos relacionados ao tipo de vegetação e altitude. Além disso, as diferenças nos métodos empregados nestes estudos também influenciam o padrão de similaridade, uma vez que apenas na E.E. Bananal foram utilizadas armadilhas de interceptação e queda. O agrupamento de S.J.Barreiro com a Serra do Japi, e não com a E.E. Bananal, por outro lado, pode ser decorrente da união das listas de espécies obtidas nas duas formações florestais amostradas, FO e FE. Esta fusão deve ter colaborado para minimizar a semelhança da taxocenose de S.J.Barreiro com a da E.E. Bananal, uma vez que esta é composta exclusivamente por remanescentes de Floresta Ombrófila Densa e de Floresta Ombrófila Mista em menor proporção. Por outro lado, a comparação da lista conjunta para as duas localidades favoreceu a semelhança da taxocenose de S.J.Barreiro com a da Serra do Japi, que se situa em área de tensão ecológica, resultante do encontro da Floresta Ombrófila Densa com a Floresta Estacional Semidecidual (Leitão Filho 1992, SP: SMA/IF 2005), e apresenta uma grande diversidade de espécies representativas de ambos os ecossistemas (Ribeiro et al. 2005).

Desta forma verifica-se que, ao menos em uma escala geográfica menor como a abrangida neste estudo, as taxocenoses de anuros com maior similaridade na composição de espécies são as de formações florestais (fisionomias) semelhantes. A correspondência desse padrão de similaridade com as fisionomias vegetais tem sido apontada em diversos estudos (Pombal Jr. \& Gordo 2004, Brasileiro et al. 2005, Conte \& Rossa-Feres 2006).

O conhecimento da anurofauna de S.J.Barreiro é ainda preliminar e, possivelmente, outras espécies de anuros serão encontradas a medi- da que novos estudos sejam realizados em diferentes áreas. Existem lacunas no esforço de coleta em diversas localidades da Mata Atlântica $\mathrm{e}$, em se tratando de animais associados à serapilheira normalmente de difícil captura, salientamos a necessidade da utilização de metodologias complementares. Sobretudo para a amostragem de espécies de reprodução explosiva ou de hábitos fossoriais, que poderiam vir a alterar o agrupamento de similaridade das taxocenoses de anuros. Há também uma carência de dados em relação aos inventários e a padronização dos métodos de amostragem, o que compromete a realização de comparações fidedignas entre as localidades.

\section{Considerações finais}

O município de São José do Barreiro possui a segunda maior área de vegetação nativa do Vale do Paraíba Paulista - 26.064 ha - o que representa 43,4\% de sua área total (SP:SMA/IF 2005), porém, vem sofrendo alterações ambientais como desmatamentos, extração seletiva de espécies vegetais e principalmente queimadas (MMA/ IBAMA 2004), como a ocorrida no mês de junho de 2006, em áreas de pastagens e de capoeiras em início de regeneração da Fazenda Atibaia (local deste estudo) e de propriedades vizinhas (H. Serafim, obs. pessoal). A grande riqueza de espécies de anuros registrada neste estudo ressalta a importância da preservação de remanescentes florestais localizados em propriedades particulares para a conservação da anurofauna. Se somarmos o inventário deste estudo ao realizado por Zaher et al. (2005), no município vizinho, Bananal, temos 47 espécies de anuros, o que coloca a região como terceira mais rica em espécies do estado de São Paulo. Portanto torna-se premente a intensificação da vigilância ambiental e a realização de estudos mais extensos que busquem conhecer a biodiversidade da região, afim de melhor subsidiar propostas que envolvam o manejo, conservação e o monitoramento da biodiversidade. Ressaltamos ainda que o Vale do Paraíba paulista não possui áreas de proteção integral que incluam remanescentes de Floresta Estacional Semidecidual, o que compromete seriamente a conservação de parte importante da biota, que é exclusiva deste tipo florestal, como constatado neste estudo para a taxocenose de anuros.

\section{Agradecimentos}

Agradecemos toda a confiança e apoio oferecidos pela Profa. Dra. Denise Maria Peccinini-Seale (in memorian), que coordenou o projeto "Diversidade e Conservação da Fauna de Répteis no Sudeste da Floresta Atlântica", vinculado ao Programa BIOTAFAPESP (Processo n ${ }^{\circ}$ 99/08291-5), que financiou este estudo; somos gratos aos proprietários da Fazenda Atibaia e da Pousada Recanto da Floresta, pela autorização e cooperação; aos companheiros de campo: F.L. Franco (IBSP), D.M. Peccinini-Seale, M.A. Sena, E.S. Cardoso e I. Rubio (IB/USP); ao Dr. Célio F.B. Haddad pelo auxílio na identificação dos anuros; ao Instituto de Biociências, USP (São Paulo, SP); ao Instituto Butantan (São Paulo, SP); RAN, IBAMA (Goiânia, GO) Proc. 02027.004018/01-26; a dois assessores anônimos pelas sugestões; ao Parque Nacional da Serra da Bocaina (SP/RJ) e ao Instituto Florestal (São Paulo, SP) Proc. 40.128. Crédito das fotografias: Fotógrafo Pepe Mélega: Figuras 2d, 2e e 2i; Fotógrafa Rita Barreto: Figura 2q; Ms. Susan Ienne: Figura 2h; as demais são de autoria do Biólogo Herbert Serafim.

\section{Referências Bibliográficas}

AMPHIBIAWEB. 2008. AmphibiaWeb: Information on amphibian biology and conservation. [web application]. Berkeley, California. http:// amphibiaweb.org/ (último acesso em 15/08/2006). 
BERTOLUCI, J. 2001. Anfíbios anuros. In Intervales, Fundação Florestal (C. Leonel, org.). Secretaria de Estado do Meio Ambiente, São Paulo, p.158-167.

BERGALLO, H.G., ROCHA, C.F.D., ALVES, M.A.S. \& SLUYS, M.V. 2000. A fauna ameaçada de extinção do estado do Rio de Janeiro. Editora da Universidade do Estado do Rio de Janeiro, Rio de Janeiro.

BRASILEIRO, C.A., SAWAYA, R.J., KIEFER, M.C. \& MARTINS, M. 2005. Amphibians of an open Cerrado fragment in southern Brazil. Biota Neotrop. 5(2): http://www.biotaneotropica.org.br/v5n2/pt/ abstract?article+BN00405022005 (último acesso em 07/11/2006).

CHAPARRO, J.C., Pramuk, J.B. \& Gluesenkamp, A.G. 2007. A new species of arboreal Rhinella (Anura: Bufonidae) from cloud forest of southeastern Peru. Herpetologica 63:203-212.

CONTE, C.E. \& ROSSA-FERES, D.C. 2006. Diversidade e ocorrência temporal da anurofauna (Anura: Amphibia) em São José dos Pinhais, Paraná, Brasil. Rev. Bras. Zool. 23(1):162-175.

DAJOZ, R. 1972. Ecologia Geral. Editora Vozes/Editora da Universidade de São Paulo, São Paulo.

DEAN, W. 1997. A ferro e fogo. A história e a devastação da Mata Atlântica brasileira. Companhia das Letras, São Paulo.

DUELLMAN, W.E. 1978. The biology of an equatorial herpetofauna in Amazonian Ecuador. Miscellaneous publication Museum Natural History University of Kansas, Lawrence, Kansas. 65:1-352.

DUELLMAN, W.E. 1988. Patterns of species diversity in anuran amphibians in the American Tropics. Ann. Missouri Bot. Gard. 75(1):79-104.

ETEROVICK, P.C., CARNAVAL, A.C.O.Q., BORGESNOJOSA, D.M., SILVANO, D.L., SEGALLA, M.V. \& SAZIMA, I. 2005. Amphibian declines in Brazil: an overview. Biotropica 37(2):166-179.

FAIVOVICH, J., HADDAD, C.B.F., GARCIA, P.C.A., FROST, D.R., CAMPBELL, J.A. \& WHEELER. W.C. 2005. Systematic review of the frog family Hylidae, with special reference to Hylinae: a phylogenetic analysis and taxonomic revision. Bull. Amer. Mus. Nat. Hist. 294:1-240.

FROST, D.R., GRANT, T., FAIVOVICH, J., BAIN, R.H., HAAS, A., HADDAD, C.F.B., AS, R.O, CHANNING, A., WILKINSON, M., DONNELLAN, S.C., RAXWORTHY, C., CAMPBELL, J.A., BLOTTO, B.L., MOLER, P., DREWES, R.C., NUSSBAUM, R.A., LYNCH, J.D., GREEN, D.M. \& WHEELER, W.C. 2006. The amphibian tree of life. Bull. Amer. Mus. Nat. Hist. 297:1-370.

FONSECA, R.C.B. \& RODRIGUES, R.R. 2000. Análise estrutural e aspectos do mosaico sucessional de uma floresta semidecídua em Botucatu, SP. Scientia Forestalis 57:27-43.

FUNDAÇÃO SOS MATA ATLÂNTICA \& INPE. 1993. Evolução dos remanescentes florestais e ecossistemas associados do domínio da Mata Atlântica. Fundação SOS Mata Atlântica e Instituto Nacional de Pesquisas Espaciais, São Paulo.

FUNDAÇÃO SOS MATA ATLÂNTICA. 1998. Atlas da evolução dos remanescentes florestais e ecossistemas associados no domínio da Mata Atlântica no período 1990-1995. Fundação SOS Mata Atlântica, São Paulo.

GRANT, T., FROST, D.R., CALDWELL, J.P., GAGLIARDO, R., HADDAD, C.F.B., KOK, P.J.R., MEANS, B.D., NOONAN, B.P., SCHARGEL, W. $\&$ WHEELER, W.C. 2006. Phylogenetic systematics of dart-poison frogs and their relatives (Amphibia: Athesphatanura: Dendrobatoidea). Bull. Amer. Mus. Nat. Hist. 299:1-262.

HADDAD, C.F.B. 1998. Biodiversidade dos anfíbios no Estado de São Paulo. In Biodiversidade do Estado de São Paulo, Brasil: síntese do conhecimento ao final do século XX (R.M.C. Castro, ed.). Editora FAPESP, São Paulo. v.6, p. 17-26.

HADDAD, C.F.B. \& PRADO, C.P.A. 2005. Reproductive modes in frogs and their unexpected diversity in the Atlantic Forest of Brazil. BioScienc. 55(3):207-217

HADDAD, C.F.B. \& SAZIMA, I. 1992. Anfíbios anuros da Serra do Japi. In História Natural da Serra do Japi: Ecologia e preservação de uma área florestal no Sudeste do Brasil (L.P.C. Morellato, ed.). Editora da Unicamp/ FAPESP, Campinas, p.188-211.

HEYER, W.R., RAND, A.S., CRUZ, C.A.G. \& PEIXOTO, O.L. 1988. Decimations, extinctions, and colonizations of frog populations in southeast Brazil and their evolutionary implications. Biotropica 20:230-235.

HEYER, W.R., RAND, A.S., CRUZ, C.A.G., PEIXOTO, O.L. \& NELSON C.E. 1990. Frogs of Boracéia. Arq. Zool. 31(4):231-410.

IBAMA. 2003. Lista nacional das espécies da fauna brasileira ameaçadas de extinção. http://www.mma.gov.br/port/sbf/fauna/index.cfm (último acesso em 21/10/2007)

IZECKSOHN, E. \& CARVALHO-SILVA, S.P. 2001. Anfíbios do Município do Rio de Janeiro. Editora Universidade Federal do Rio de Janeiro, Rio de Janeiro, Brasil.

JIM, J. 1980. Aspectos ecológicos dos anfíbios registrados na região de Botucatu, São Paulo (Amphibia, Anura). Tese de Doutorado, Universidade de São Paulo, São Paulo.

KREBS, C.J. 1999. Ecological Methodology. 2nd ed. Benjamin Cummings, Menlo Park, California, 620p.

LEITÃO-FILHO, H.F. 1982. Aspectos taxonômicos das florestas do estado de São Paulo. Silvicultura em São Paulo 16A:197-206.

LEITÃO-FILHO, H.F. 1992. A flora arbórea da Serra do Japi. In História natural da Serra do Japi: Ecologia e preservação de uma área florestal no Sudeste de Brasil (L.P.C. Morellato, org.). Editora da Unicamp, Campinas, 4062p.

MACHADO, A.B.M., FONSECA, G.A.B., MACHADO, R.B., AGUIAR, L.M.S. \& LINS, L.V. 1998. Livro vermelho das espécies ameaçadas de extinção da fauna de Minas Gerais. Fundação Biodiversitas, Belo Horizonte. http://www.biodiversitas.org.br/f_ameaca/index_lista.htm (último acesso: 18/09/2004).

MARTINS, S.V., PELLEGRINI, M.C. \& MARANGON, L.C. 2002. Composição florística e estrutura de uma floresta secundária no município de Cruzeiro, SP. Revista Árvore 26(1):35-41.

MITTERMEIER, R.A., GIL, P.R. \& MITTERMEIER, C.G. 1997. Megadiversity: Earth's Biologically Wealthiest Nations. Cemex, México.

MMA/IBAMA, MINISTÉRIO DO MEIO AMBIENTE. 2004. Plano de Manejo do Parque Nacional da Serra da Bocaina. Relatório Técnico. IBAMA, Brasília.

MORAES, R.A., SAWAYA, R.J. \& BARRELA, W. 2007. Composição e diversidade de anfíbios anuros em dois ambientes de Mata Atlântica no Parque Estadual Carlos Botelho, São Paulo, sudeste do Brasil. Biota Neotrop. 7(2): http://www.biotaneotropica.org.br/v7n2/pt/ abstract?article+BN00307022007 (último acesso em 29/08/2007).

MORELLATO, L.P.C., RODRIGUES, R.R., LEITÃO-FILHO, H.F. \& JOLY, C.A. 1989. Estudo comparativo da fenologia de espécies arbóreas de floresta de altitude e floresta mesófila semidecídua na Serra do Japi, Jundiaí, São Paulo. Rev. bras. Bot. 12:85-98.

MORELLATO, L.P.C. \& LEITÃO-FILHO, H.F. 1992. Padrões de frutificação e dispersão na serra do Japi. In História natural da Serra do Japi: Ecologia e preservação de uma área florestal no sudeste do Brasil. (L.P.C. Morellato, org.). Editora da UNICAMP/FAPESP, Campinas, p. 112-141.

MORELLATO, L.P.C., TALORA, D.C., TAKAHASI, A., BENCKE, C.C., ROMERA, E.C. \& ZIPARRO, V.B. 2000. Phenology of Atlantic Rain Forest Trees: A Comparative study. Biotropica 32(4b):811-823.

MYERS, N., MITTERMIER, R.A., MITTERMIER, C.G., FONSECA, G.A.B \& KENT, J. 2000. Biodiversity hotspot for conservation priorities. Nature 403:845-853.

PAPP, M.G. \& PAPP, C.O.G. 2000. Decline in a population of the treefrog Phyllodytes luteolus after fire. Herpetol. Rev. 31(2):93-95.

POMBAL-JR., J.P. 1995. Biologia reprodutiva de anuros (Amphibia) associados a uma poça permanente na Serra de Paranapiacaba, sudeste do Brasil. Tese de doutorado, Universidade Estadual Paulista, Rio Claro, SP. 
POMBAL-JR., J.P. \& HADDAD, C.F.B. 1999. Frogs of the genus Paratelmatobius (Anura: Leptodactylidae) with descriptions of two new species. Copeia 1999(4):1014-1026.

POMBAL-JR., J.P. \& GORDO, M. 2004. Anfíbios anuros da Juréia. In Estação Ecológica Juréia-Itatins. Ambiente físico, flora e fauna (O.A.V. Marques \& W. Duleba, eds). Holos editora, Ribeirão Preto, p.243-256.

RIBEIRO, R.S., EGITO, G.T.B.T. \& HADDAD, C.F.B. 2005. Chave de identificação: anfíbios anuros da vertente de Jundiaí da Serra do Japi, Estado de São Paulo. Biota Neotrop. 5(2): http://www.biotaneotropica. org.br/v5n2/pt/abstract?identification-key+bn03005022005 (último acesso em 25/03/2006).

ROSSA-FERES, D.C. \& JIM, J. 1994. Distribuição sazonal em comunidade de anfíbios anuros na região de Botucatu, São Paulo. Rev. Bras. Biol. 54(2):323-334

SMA-CED - SECRETARIA DO MEIO AMBIENTE DO ESTADO 1998. Fauna Ameaçada no estado de São Paulo. Série PROBIO/SP, São Paulo.

SECRETARIA DO MEIO AMBIENTE / INSTITUTO FLORESTAL. 2005 Inventário Florestal da Vegetação Natural do Estado de São Paulo. Imprensa oficial, São Paulo.

SBH. 2008. Lista de espécies de anfíbios do Brasil. Sociedade Brasileira de Herpetologia (SBH). http://www.mma.gov.br/port/sbf/fauna/index.cfm (último acesso em 07/01/2008).

SILVANO, D.L. \& SEGALLA, M.V. 2005. Conservação de anfíbios no Brasil. Megadiversidade 1(1):79-86.
TABARELLI, M., PINTO, L.P., SILVA, J.M.C., HIROTA, M.M. \& BEDÊ, L.C. 2005. Desafios e oportunidades para a conservação da biodiversidade na Mata Atlântica brasileira. Megadiversidade 1(1):132-138.

VASCONCELOS, P.C.S. 1992. Fitossociologia de uma vegetação em sucessão secundária no Vale do Paraíba, São Paulo. Dissertação de Mestrado, Universidade Federal de Viçosa, Minas Gerais.

VASCONCELOS, T.S. \& ROSSA-FERES. D.C. 2005. Diversidade, distribuição espacial e temporal de anfíbios anuros (Amphibia, Anura) na região noroeste do estado de São Paulo, Brasil. Biota Neotrop. 5(2): http:// www.biotaneotropica.org.br/v5n2/pt/abstract?article+BN01705022005 (último acesso em 21/10/2007).

VELOSO, H.P., RANGEL-FILHO, A.L.R. \& LIMA, J.C.A. 1991. Classificação da vegetação brasileira, adaptada a um sistema universal. IBGE, Departamento de Recursos Naturais e Estudos Ambientais, Rio de Janeiro.

ZAHER, H., AGUIAR, E. \& POMBAL-JR., J.P. 2005. Paratelmatobius gaigeae (Cochran 1938) re-discovered (Amphibia, Anura, Leptodactylidae). Arq. Mus. Nac. 63(2):321-328.

YOUNG, B., LIPS, K.R., REASER, J.K., IBAÑEZ, R., SALAS, A.W., CEDEÑO, J.R., COLOMA, L.A., RON, S., LA MARCA, E., MEYER, J. R., MUÑOZ, A., BOLAÑOS, F., CHAVES, G. \& ROMO, D. 2001. Population declines and priorities for Amphibian conservation in Latin America. Conserv. Biol. 15(5):1213-1223.

Recebido em 22/10/07 Versão Reformulada recebida em 10/04/08 Publicado em 17/04/08 